\title{
A NOVAL WAY OF CLIENT DATA STORAGE IN CLOUd USING TPA SECURITY
}

\author{
B. Radha Madhavi ${ }^{1}$, Dr Jammi Ashok ${ }^{2} \&$ Dr. Sateesh Nagavarapu ${ }^{3}$
}

\begin{abstract}
Key betrayal perpetually has always been an critical issue for in-depth cyber defence in lots of protection programs. Recently, the way to prearrangement with the important thing publicity trouble within the settings of cloud garage auditing has been contemplated and studied. To cope with the project, current answers all require the consumer to update his secret keys in whenever period, which may additionally inevitably bring in new neighborhood hindrance to the purchaser, mainly people with constrained computation resources, such as cell phones. In this paper, we cynosure on the way to make the key updates as transparent as feasible for the customer and recommend a new paradigm referred to as cloud garage auditing with verifiable outsourcing of key updates. In this paradigm, key updates may be competently outsourced to a few legal birthday celebration, and hence the important thing-replace burden at the purchaser could be kept minimum. In precise, we leverage the 0.33 party auditor (TPA) in many present public auditing designs, let it play the role of authorized celebration in our case, and make it in fee of each the garage auditing and the comfy key updates for key-publicity resistance. In our layout, TPA simplest desires to hold an encrypted version of the client's secret key at the same time as doing a lot of these burdensome obligations on behalf of the customer. The customer handiest needs to download the encrypted secret key from the TPA while uploading new files to cloud. Besides, our design also equips the client with functionality to in addition verify the validity of the encrypted mystery keys furnished by the TPA. All these salient capabilities are cautiously designed to make the whole auditing system with key exposure resistance as obvious as viable for the purchaser. We formalize the definition and the safety version of this paradigm. The safety evidence and the performance simulation display that our targeted design instantiations are relaxed and efficient.
\end{abstract}

Keywords: Cloud Storage, Client, TPA, Time Stamp

\section{INTRODUCTION}

Appropriated figuring is the use of enrolling resources (hardware and programming) that are passed on as an organization over a framework (routinely the Internet). The name starts from the normal use of a cloud-shaped picture as a consultation for the brain boggling establishment it contains in system outlines. Disseminated processing supplies remote organizations with a customer's data, programming and computation. Appropriated processing involves gear and programming resources made available on the Internet as supervised pariah organizations. These organizations consistently offer access to bleeding edge programming applications and awesome frameworks of server PCs.

The objective of distributed computing is to apply customary supercomputing, or superior registering power, regularly utilized by military and research offices, to perform several trillions of calculations for every second, in customer situated applications, for example, budgetary portfolios, to convey customized data, to give information stockpiling or to control vast, immersive PC diversions. The distributed computing utilizes systems of expansive gatherings of servers ordinarily running minimal effort shopper PC innovation with specific associations with spread information preparing errands crosswise over them. This mutual IT foundation contains vast pools of frameworks that are connected together. Regularly, virtualization strategies are utilized to expand the energy of distributed computing.

\subsection{Characteristics and Services Models}

Broad arrange get to: Capabilities are accessible over the system and got to through standard instruments that advance use by heterogeneous thin or thick customer stages (e.g., cell phones, portable workstations, and PDAs).

Resource pooling: The supplier's registering assets are pooled to serve various shoppers utilizing a multi-occupant display, with various physical and virtual assets progressively alloted and reassigned by buyer request. There is a feeling of area freedom in that the client by and large has no control or learning over the correct area of the gave assets yet might have the capacity to determine area at a larger amount of deliberation (e.g., nation, state, or server farm). Cases of assets incorporate capacity, handling, memory, organize data transmission, and virtual machines.

\footnotetext{
${ }^{1}$ M.Tech CSE,Malla Reddy Institute of Technology, Hyderabad

${ }^{2}$ Principal, Malla Reddy Institute of Technology, Hyderabad

${ }^{3}$ Associate Professor, Dept. of CSE, Malla Reddy Institute of Technology
} 
Rapid versatility: Capabilities can be quickly and flexibly provisioned, now and again naturally, to rapidly scale out and quickly discharged to rapidly scale in. To the purchaser, the abilities accessible for provisioning frequently give off an impression of being boundless and can be acquired in any amount whenever.

Measured benefit: Cloud frameworks naturally control and streamline asset use by utilizing a metering ability at some level of reflection suitable to the sort of administration (e.g., capacity, handling, transfer speed, and dynamic client accounts). Asset utilization can be overseen, controlled, and revealed giving straightforwardness to both the supplier and purchaser of the used administration.

\subsection{Characteristics of cloud computing}

Distributed computing includes three diverse administration models, to be specific Infrastructure-as-a-Service (IaaS), Platform-as-a-Service (PaaS), and Software-as-a-Service (SaaS). The three administration models or layer are finished by an end client layer that epitomizes the end client point of view on cloud administrations. In the event that a cloud client gets to administrations on the foundation layer, for example, she can run her own particular applications on the assets of a cloud framework and stay in charge of the help, upkeep, and security of these applications herself. On the off chance that she gets to an administration on the application layer, these undertakings are regularly dealt with by the cloud specialist organization.

\subsection{Benefits of cloud computing}

Achieve economies of scale - increment volume yield or efficiency with less individuals. Your cost per unit, undertaking or item plunges.

Reduce spending on innovation framework. Keep up simple access to your data with negligible forthright spending. Pay as you go (week by week, quarterly or yearly), in view of interest.

Globalize your workforce for next to nothing. Individuals worldwide can get to the cloud, if they have an Internet association.

\section{LITERATURE SURVEY}

2.1 Secure outsourcing of logical calculations

Creators: M. J. Atallah, K. N. Pantazopoulos, J. R. Rice, and E. E. Spafford

We examine the outsourcing of numerical and logical calculations utilizing the accompanying structure: A client who needs calculations done yet does not have the computational assets (processing power, suitable programming, or programming ability) to do these locally, might want to utilize an outer operator to play out these calculations. This as of now emerges in numerous handy circumstances, including the money related administrations and oil administrations businesses. The outsourcing is secure in the event that it is managed without uncovering to the outside specialist either the real information or the genuine response to the calculations. The general thought is for the client to do some painstakingly planned neighborhood preprocessing (masking) of the issue as well as information before sending it to the specialist, and furthermore some nearby postprocessing of the appropriate response came back to remove the truse reply. The mask procedure ought to be as lightweight as could be allowed, e.g., require some serious energy relative to the span of the information and reply. The camouflage preprocessing that that the client performs locally to "conceal" the genuine calculation can change the numerical properties of the computational performance. We exhibit a framewrok for camouflaging logical copmutations and talk about their costs, numerical properties, and levels of security. These mask procedures can be inserted in an abnormal state, simple to-utilize framework (critical thinking condition) that shrouds their intricacy.

\subsection{Private and swindling free outsourcing of mathematical calculations}

Creators: D. Benjamin and M. J. Atallah

We give conventions for the protected and private outsourcing of straight variable based math calculations, that empower a customer to safely outsource costly arithmetical calculations (like the augmentation of gigantic grids) to two remote servers, to such an extent that the servers get the hang of nothing about the client's private info or the consequence of the computation,and any endeavored defilement of the appropriate response by the servers is identified with high likelihood. The computational work done locally by the customer is direct in the measure of its info and does not require the customer to complete locally any costly encryptions of such input.The computational weight on the servers is corresponding to the time many-sided quality of the current for all intents and purposes utilized calculations for taking care of the arithmetical issue (e.g., relative to $\mathrm{n}^{\wedge} 3$ for increasing two $\mathrm{n} \times \mathrm{n}$ lattices). On the off chance that the servers were to plot against the client,then they would just discover the customer's private information sources, however they would not have the capacity to degenerate the appropriate response without recognition by the customer.

\subsection{Secure and reasonable outsourcing of straight programming in distributed computing}

Creators: C. Wang, K. Ren, and J. Wang

Distributed computing empowers clients with restricted computational assets to outsource extensive scale computational errands to the cloud, where enormous computational power can be effectively used in a compensation for every utilization way. In any case, security is the real worry that keeps the wide appropriation of calculation outsourcing in the cloud, particularly when end-client's private information are prepared and delivered amid the calculation. Along these lines, secure 
outsourcing components are in incredible need to not just ensure delicate data by empowering calculations with encoded information, yet in addition shield clients from malignant practices by approving the calculation result. Such a system of general secure calculation outsourcing was as of late appeared to be achievable in principle, yet to outline components that are for all intents and purposes productive remains an exceptionally difficult issue. Concentrating on building figuring and improvement errands, this paper examines secure outsourcing of generally material direct programming (LP) calculations. With a specific end goal to accomplish down to earth effectiveness, our system configuration unequivocally deteriorates the LP calculation outsourcing into open LP solvers running on the cloud and private LP parameters claimed by the client. The subsequent adaptability enables us to investigate fitting security/productivity tradeoff by means of more elevated amount reflection of LP calculations than the general circuit portrayal. Specifically, by planning private information claimed by the client for LP issue as an arrangement of grids and vectors, we can build up an arrangement of effective security saving issue change systems, which enable clients to change unique LP issue into some irregular one while ensuring touchy information/yield data. To approve the calculation result, we additionally investigate the central duality hypothesis of LP calculation and infer the important and adequate conditions that right outcome must fulfill. Such outcome check system is greatly effective and brings about near zero extra cost on both cloud server and clients. Broad security examination and test comes about demonstrate the prompt practicability of our component plan.

\section{EXISTING SYSTEM}

In Present machine a distributed storage evaluating convention with key-introduction versatility by methods for refreshing the client's mystery keys occasionally. In this way, the harm of key introduction in cloud carport inspecting can be diminished. Be that as it may, it also gets new neighborhood loads for the customer in light of the fact that the benefactor needs to execute the vital thing refresh calculation in at whatever point period to influence his mystery key to course ahead. For a few clients with limited calculation resources, they dislike doing such more calculations by methods for themselves in at whatever point period. It would be obviously all the more speaking to make key updates as straightforward as feasible for the customer, for the most part in like manner key supplant consequences. Wang et al. Proposed an open security holding examining convention. They utilized the irregular ensuring way to deal with influence the convention to acquire privateness keeping property.

\section{PROPOSED SYSTEM}

In our proposed machine another worldview alluded to as cloud carport evaluating with unquestionable outsourcing of key updates. In this new worldview, key-supplant operations aren't proficient by method for the client, however by methods for an authorized birthday festivity. The lawful birthday party holds a scrambled riddle key of the benefactor for distributed storage evaluating and refreshes it underneath the encoded nation in on each event length. The benefactor downloads the encoded mystery key from the approved birthday party and unscrambles it best while he might truly want to add new reports to cloud. What's more, the client can affirm the legitimacy of the scrambled mystery key. We format the principal cloud carport examining convention with unquestionable outsourcing of key updates. In our plan, the third birthday celebration party evaluator (TPA) plays out the capacity of the approved festival who is responsible for key updates. We formalize the definition and the security variant of the distributed storage reviewing convention with undeniable outsourcing of key updates. We furthermore demonstrate the wellbeing of our convention inside the formalized security display and legitimize its execution by method for solid usage.

\section{IMPLEMENTATION \& RESULTS}

Customer Module :This module incorporates the Client enrollment and customer login data, Every Client need to enlist while accessing to the cloud, Every Client may be actuated by means of the Cloud, After Cloud enacted, every Client need to give time stamp add key to transfer a fresh out of the plastic new records into cloud, Time stamp transfer key will be outfitted by outsider examiner, Client need to download the time stamp include key while benefactor bringing in new archives into cloud, Client can see document information and download the record utilizing time stamp document key gave by utilizing TPA.

Time stamp include key: Time stamp include key can be outfitted by methods for TPA. Customer can down load the include key each time buyer bringing in new record into cloud and that they require never again to offer demand key from TPA, At the season of client downloading the time stamp transfer key, the demand will deliver in quickly to TPA and supplant steady with time with the guide of TPA and send scrambled transfer riddle key to purchaser, And at last, benefactor can unscramble down load the include mystery key, After getting decode include puzzle key, now Client can include a spic and span document into cloud.

Time stamp report key: Each time customer accessing and downloading the archive from cloud, TPA will give each time record refresh key to customer enlisted mail Id. So same archive key won't be there for approach record, It will transport as report time stamp refresh key, so relating customer can utilize this record from particular server with no other utilization of programmer or aggressor, If Client again login with same server or diverse server, rise to report key won't been used by Client to download the record for greater security.

Outsider Auditor (TPA) Module : It goes about as administrator, TPA Provide time Upload riddle enter in Encrypted state for every shopper to include new record into cloud. It can be deliver as in quickly even as Client downloading the include key, 
The include secret key, while client downloading key it will up and coming predictable with time, After cloud given reviewing proof at that point best TPA can review all records, And additionally offer the File Stamp key for all records to the client ask for comparing reports key.

Cloud Module : Actuate data customer, Cloud sends carport inspecting proof for all records to TPA, Cloud can see the buyer downloaded archives from cloud.

\subsection{Comparision between Existing and Proposed System:}

Existing System:

Existing framework doesn't care for reviewing convention with irrefutable outsourcing of key updates.

- Third birthday festivity has the entrance to peer buyer's mystery key without encryption.

- No confirmation framework accessible for buyer's for to check legitimacy of the scrambled secret keys while downloading them from the TPA

- All display examining conventions are altogether based on the conviction that the mystery key of the client is really calm and could now not be uncovered.

Proposed System:

- The TPA does never again know the genuine mystery key of the supporter for distributed storage evaluating, however handiest holds a scrambled model. In the unmistakable convention, we utilize the blinding strategy with holomorphic property to shape the encryption calculation to scramble the name of the amusement keys held by means of the TPA. It makes our convention loose and the unscrambling operation green.

- In the interim, the TPA can finish key updates underneath the encoded state. The buyer can confirm the legitimacy of the encoded puzzle key while he recovers it from the TPA. The buyer downloads the scrambled mystery key from the approved birthday party and unscrambles it best when he might truly want to add new archives to cloud. Furthermore, the purchaser can check the legitimacy of the scrambled mystery key.

- Distributed storage evaluating convention with obvious outsourcing of key updates The customer can confirm the legitimacy of the encoded mystery key when he recovers it from the TPA The insurance model of the distributed storage examining convention with certain outsourcing of key updates.

\section{RESULTS:}

Step1: Client Login into application

Step2: If user wants to download a file then he will get a time stamp upload key and then user will decrypt and download it Step3: By using this user can download the file he wanted. By this no third party can hack the data while he is downloading. Step4: If user wants to upload file he can upload directly to cloud then for that particular file a TPA will be generated to download.

Step5: Data will be stored in cloud and files will be sent by TPA. Here TPA plays a major role in retrieving data in secure manner.

Step6: Here admin can accept or reject user to upload or download file. Without his permission user cannot do any operations.

\section{CONCLUSION}

In this paper, we take a gander at while in transit to outsource key updates for cloud carport reviewing with scratch introduction versatility. We prompt the principal distributed storage examining convention with unquestionable outsourcing of key updates. In this convention, key updates are outsourced to the TPA and are straightforward for the customer. What's more, the TPA just observes the scrambled model of the buyer's puzzle key, while the buyer can correspondingly check the legitimacy of the encoded riddle keys while downloading them from the TPA. We convey the formal assurance confirm and the execution reproduction of the proposed conspire.

\section{REFERENCES}

[1] [1] Jia Yu, Kui Ren, Fellow, IEEE, and Cong Wang, Member, IEEE "Empowering Cloud Storage Auditing With Verifiable Outsourcing of Key Updates " IEEE TRANSACTIONS ON INFORMATION FORENSICS AND SECURITY, VOL. 11, NO. 6, JUNE 2016

[2] [2] D. Benjamin and M. J. Atallah, "Private and duping free outsourcing of arithmetical calculations," in Proc. sixth Annu. Conf. Protection, Secur. Confide in, 2008, pp. 240-245.

[3] [3] C. Wang, K. Ren, and J. Wang, "Secure and viable outsourcing of direct programming in distributed computing," in Proc. IEEE INFOCOM, Apr. 2011, pp. 820-828.

[4] [4] X. Chen, J. Li, J. Mama, Q. Tang, and W. Lou, "New calculations for secure outsourcing of secluded exponentiations," in Proc. seventeenth Eur. Symp. Res. Comput. Secur., 2012, pp. 541- 556.

[5] [5] G. Ateniese et al., "Provable information ownership at untrusted stores," in Proc. fourteenth ACM Conf. Comput. Commun. Secur., 2007, pp. 598609.

[6] [6] A. Juels and B. S. Kaliski, Jr., "PORs: Proofs of retrievability for huge documents," in Proc. fourteenth ACM Conf. Comput. Commun. Secur., 2007, pp. 584- 597. 
[7] [7] H. Shacham and B. Waters, "Minimized evidences of retrievability," in Advances in Cryptology. Berlin, Germany: Springer-Verlag, 2008, pp. 90107.

[8] [8] G. Ateniese, R. Di Pietro, L. V. Mancini, and G. Tsudik, "Adaptable and effective provable information ownership," in Proc. fourth Int. Conf. Secur. Security Commun. Netw., 2008, Art. ID 9.

[9] [9] J. Yu, K. Ren, C. Wang, and V. Varadharajan, "Empowering distributed storage evaluating with key-presentation resistance," IEEE Trans. Inf. Legal sciences Security, vol. 10, no. 6, pp. 1167-1179, Jun. 2015.

[10] [10] D. Chaum and T. Pedersen, "Wallet databases with spectators," in Advances in Cryptology. Berlin, Germany: Spr 